\title{
A PEDAGOGICAL MODEL FOR TEACHING SCIENTIFIC DOMAIN KNOWLEDGE
}

\author{
Barry Kort ${ }^{1}$, Rob Reilly ${ }^{2}$
}

\begin{abstract}
Numerous research studies support the claim that affect plays a critical role in decision-making and performance as it influences cognitive processes. Despite this body of research there is insufficient theory within educational pedagogy to recognize and address the role and function of affect. The innovative models and theories that have been proposed to facilitate advancement in the field of educational pedagogy tend to focus on cognitive factors. Consequently, affective cues, which have a significant role, are often misinterpreted or ignored. We propose several new models for framing a dialogue leading to new insights and innovations that incorporate theories of affect into educational pedagogy.
\end{abstract}

Index Terms - Affect, emotions, model-based reasoning.

\section{INTRODUCTION}

Seasoned educators have long been aware of the importance of affect (or emotions) in learning [1]. Nonetheless there has been little in the way of comprehensive theory characterizing the relationship of emotion(s) to learning. We introduce a novel model that yields insight into this relationship.

To understand why such a model is needed let us first examine the current educational model. The current model, as shown in Figure 1, begins with 'data,' which is a collection of answers to questions that the learner has not yet seen fit to ask or needed to ask. Such data becomes 'information' when it answers a question that the learner cares to ask. For the most part, a teacher, who must somehow motivate the student to care enough to seek the answers found in the data, supplies these questions. Studying is like 'panning for gold' where the answers are the 'nuggets' buried in a ton of otherwise uninteresting gravel. Once we have our 'nuggets of information' how do we organize them into a 'body of knowledge'? We may think of 'information' as the pieces of an unassembled jigsaw puzzle, whereas 'knowledge' is the assembled jigsaw puzzle. That is, the question-answer pairs are organized into a coherent structure, in the logical and natural order in which new questions arise as soon as old ones are answered.

The assembled 'jigsaw puzzle of knowledge' reveals a previously hidden picture-a 'big picture,' if you will. Or to put it another way, the assembled 'jigsaw puzzle of knowledge' is a tapestry into which is woven many otherwise hidden and previously unrevealed stories.
The novel model shown below in Figure 2 goes beyond the current model shown in Figure 1. The focii of attention shifts to the construction of 'knowledge' and to the extraction of meaningful 'insights' from the 'big picture.' When 'knowledge' is coupled with an appropriate personal or cultural value system, 'wisdom' emerges. In other words, wisdom allows us to harness the power of knowledge for beneficial purposes. (Of course, one can adopt an inappropriate value system yielding foolishness rather than wisdom.)

'Wisdom' affords us the possibility of extracting the stories woven into the tapestry of knowledge. So from 'wisdom' we craft the bardic arts of story making and story telling. The ancients crafted myths and legends. These were the prototypical stories of their cultures, which were intended to impart 'wisdom.' A story is thus an anecdote drawn from the culture. A well-crafted anecdote or story has value both as an amusement and as a source of insight into the world from which it is drawn. And the plural of 'anecdote' is data-a collection of anecdotal stories or evidence. This observation closes the loop in Figure 2.

Figure 2 suggests a novel model that, on a fundamental level, supports an improved educational pedagogy. This will serve as a foundation for the next part of our model-how a learner's affective state should be incorporated into the overall model. Notice that the lower half of Figure 2 corresponds to the sciences while the upper-half corresponds to the humanities.

\section{THE ENTERTAINMENT-DRAMA MODEL}

Traditionally stories have been an important vehicle for the transmission of cultural knowledge. Here we outline a basic model of story craft and drama to bridge the middle portion of the chain of linked economies and to close the loop between science and the humanities in Figure 2. To this end we envision a Theory of Drama as an extension of Game Theory.

What distinguishes Drama from Game Theory is that the participants in a drama are driven by a more general collection of motivating factors than arise in a structured game. We can model the characters in a drama in terms of their Psychology, which comprises five elements: Fears, Emotions, Beliefs, Desires, and Intentions. Unlike a game where the intentions of the players are universally understood, the Psychology of the characters in drama may be deeply hidden both from the audience and from the characters themselves. The drama makes sense when one

\footnotetext{
${ }^{1}$ Barry Kort Ph.D., Media Laboratory, M.I.T., 20 Ames Street (E15-020), Cambridge, MA 02139 bkort@media.mit.edu

${ }^{2}$ Rob Reilly Ed.D., Media Laboratory, M.I.T., reilly@ media.mit .edu

0-7803-7444-4/02/\$17.00 @ 2002 IEEE

November 6 - 9, 2002, Boston, MA 


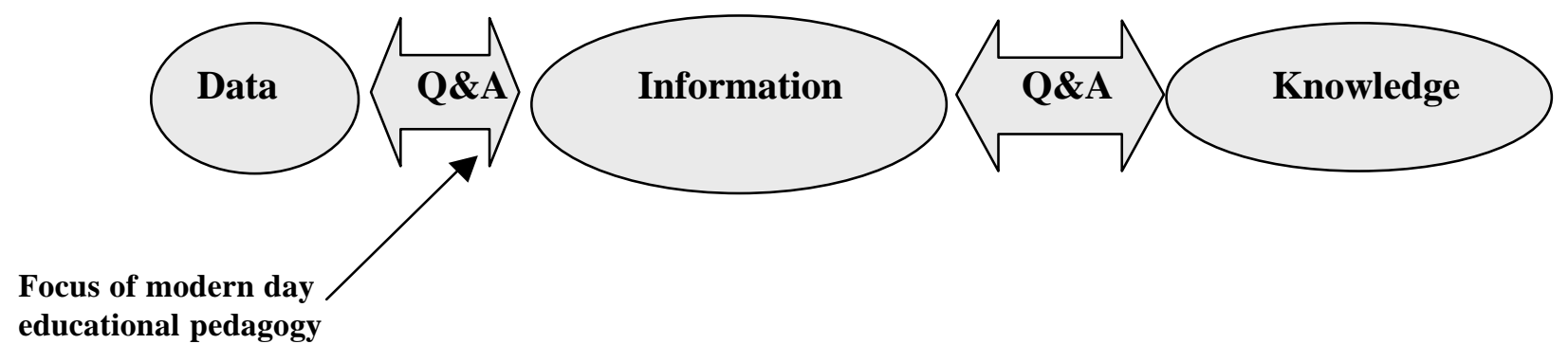

FIGURE. 1

OLD MODEL: SUPPORTS RULE-BASED LEARNING.

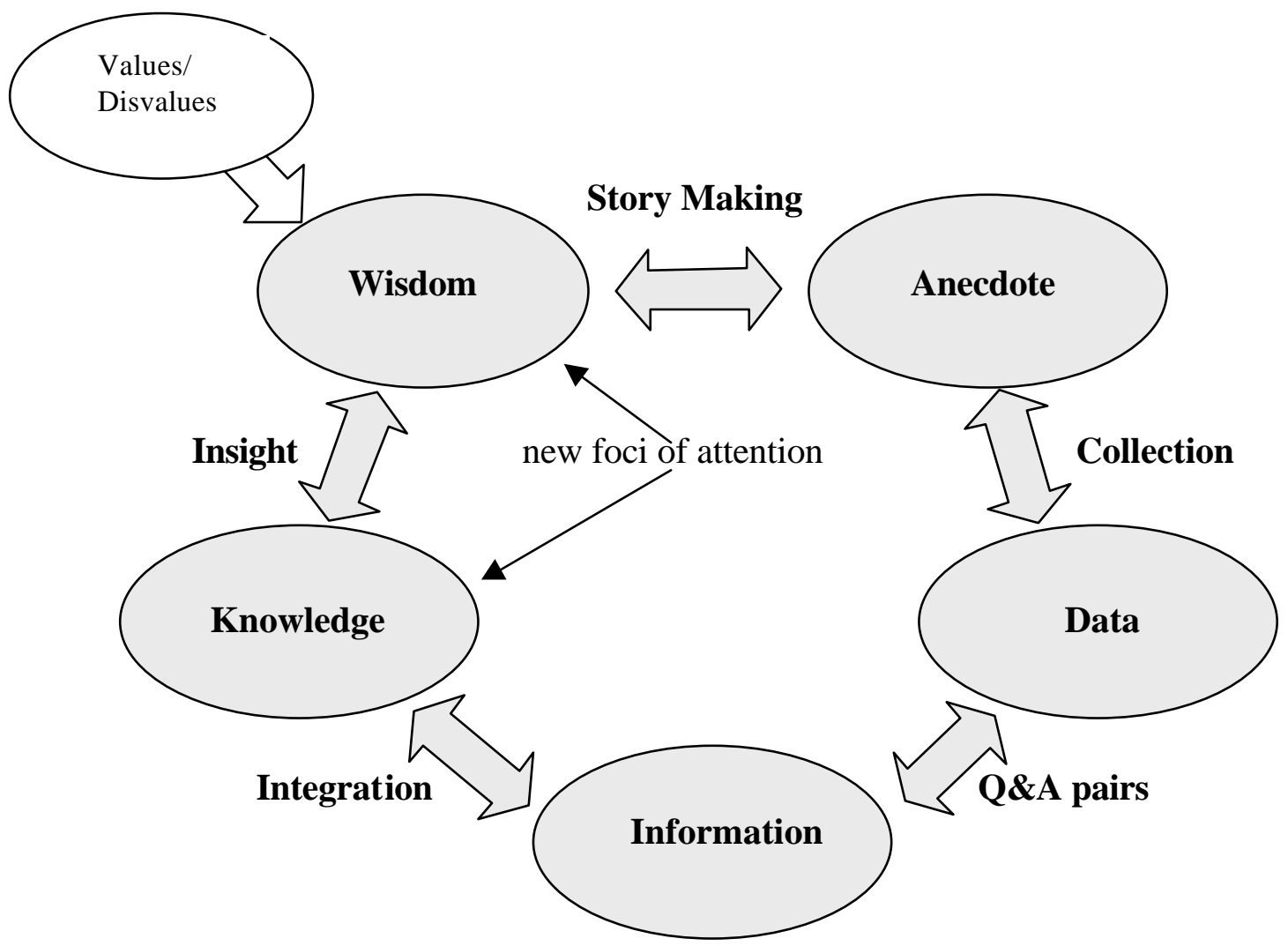

FIGURE. 2

NEW MODEL: SUPPORTS MODEL-BASED REASONING.

fills in the blanks characterizing the underlying Psychology of each player. Key dramatic moments correspond to BeliefCrystallizing events, Belief-Confirming events, and BeliefShattering events. The basic tension and conflict arise from competing desires and intentions driven by hidden emotional factors and dissimilar beliefs. As the drama unfolds, the characters must confront the realities that conflict with their Psychology and arrive at a resolution. Thus Drama is both entertaining and educational, emotionally affecting and enlightening. Modern classroom education rarely rises to the level of intrigue found in good stories. Nonetheless, classroom learning is not without some emotional component, which we will explore in the next section.

\section{MODELS OF EMOTIONS AND LEARNING}

In an attempt to install/build/re -engineer the current state of educational pedagogy educators should first look to expert teachers who are adept at recognizing the emotional state of learners, and, based upon their observations, take some action that scaffolds learning in a positive manner. But what do these expert teachers see and how do they decide upon a course of action? How do students who have strayed from learning return to a productive path, such as the one that Csikszentmihalyi [2] refers to as the "zone of flow"? This notion that a student's affective (emotional) state 


\begin{tabular}{lllllll}
\multicolumn{1}{c}{ Axis } & $\mathbf{- 1 . 0}$ & $\mathbf{- 0 . 5}$ & & $\mathbf{0}$ & $\mathbf{+ 0 . 5}$ & $\mathbf{+ 1 . 0}$ \\
Anxiety-Confidence & Anxiety & Worry & Discomfort & Comfort & Hopefulness & Confidence \\
Ennui-Fascination & Ennui & Boredom & Indifference & Interest & Curiosity & Fascination \\
Frustration-Euphoria & Frustration & Puzzlement & Confusion & Insight & Enlightenment & Euphoria \\
Dispirited-Enthusiasm & Dispirited & Disappointed & Dissatisfied & Satisfied & Thrilled & Enthusiasm \\
Terror-Excitement & Terror & Dread & Apprehension & Calm & Anticipatory & Excitement \\
Humiliation-Pride & Humiliated & Embarrassed & Self-conscious & Pleased & Satisfied & Proud
\end{tabular}

FIGURE. 3

EMOTION SETS POSSIBLY RELEVANT TO LEARNING.

\section{CONSTRUCTIVE LEARNING}

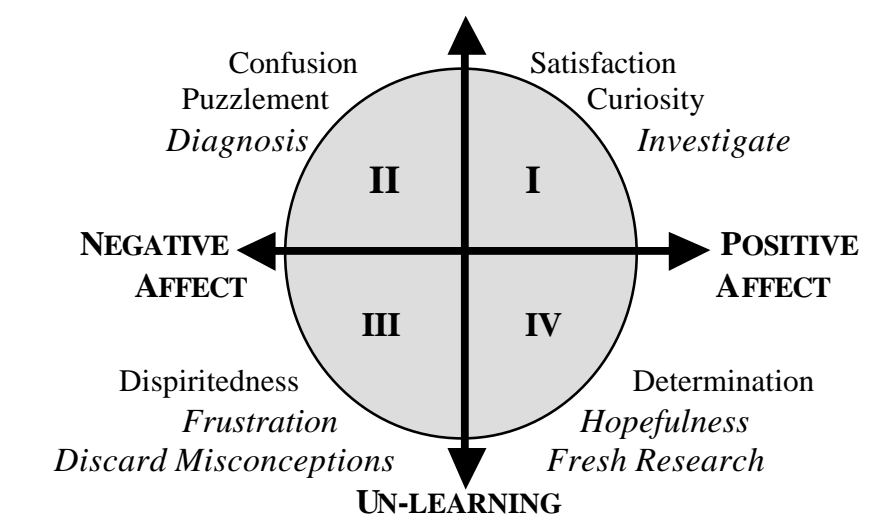

FIGURE. 4A

FOUR QUADRANT MODEL RELATING PHASES OF LEARNING TO EMOTIONS INFIGURE4.

impacts learning and that appropriate intervention based upon that affective state would facilitate earning is the concept that we propose to explore in-depth.

To prove our point, note that skilled humans can assess emotional signals with varying degrees of precision. For example, researchers are beginning to make progress giving computers similar abilities to accurately recognize affective expressions [3, 4], facial expressions [5, 6, 7, 8, 9, 10], and gestural expression [11, 12]. Although computers only perform as well as people in highly restricted domains, we believe that: a. accurately identifying a learner's cognitiveemotive state is a critical observation that will enable teachers to provide learners with an efficient and pleasurable learning experience, and, b. unobtrusive highly accurate technology will be developed to accurately assess actions in less restricted domains [see e.g., 13].

Our own preliminary pilot studies with elementary school children suggest that a human observer can assess the affective emotional state of a student with reasonable reliability based on observation of facial exp ressions, gross body language, and the content and tone of speech. If the human observer is also acting in the role of coach or mentor,

0-7803-7444-4/02/\$17.00 @ 2002 IEEE these assessments can be confirmed or refined by direct conversation (e.g. simply asking the student if she is confused or frustrated before offering to provide coaching or hints). Moreover, successful learning is frequently marked by an unmistakable elation, often jointly celebrated with "high fives." In some cases, the "Aha!" moment is so dramatic, it verges on the epiphanetic. One of the great joys for an educator is to bring a student to such a moment of triumph. But how can all educators acquire the same level of proficiency as gifted coaches, mentors, and teachers?

Our first step is to offer a model of a learning cycle, which integrates affect. Figure 3 suggests six possible emotion axes that may arise in the course of learning. Figure 4 interweaves the emotion axes shown in Figure 3 with the cognitive dynamics of the learning process. In Figure 4, the positive valence (more pleasurable) emotions are on the right; the negative valence (more unpleasant) emotions are on the left. The vertical axis is what we call the Learning Axis, and symbolizes the construction of knowledge upward, and the discarding of misconceptions downward.

November 6 - 9, 2002, Boston, MA 


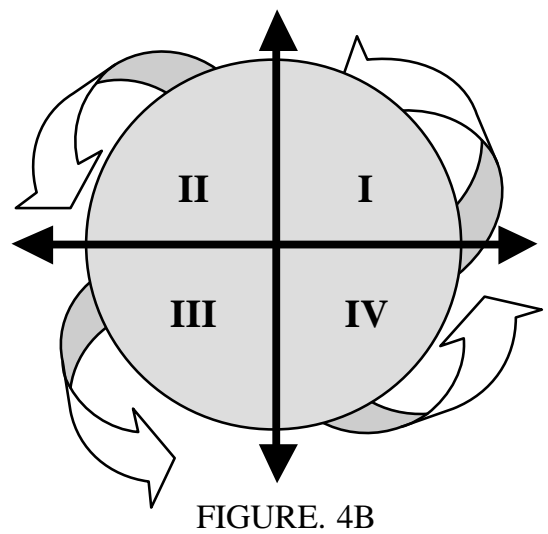

CIRCULAR AND HELICALFLOW OF EMOTION IN THE FOUR-QUADRANT MODEL.

Students ideally begin in Quadrant I or II: they might be curious or fascinated about a new topic of interest (Quadrant I) or they might be puzzled and motivated to reduce confusion (Quadrant II). In either case, they are in the top half of the space if their focus is on constructing or testing knowledge. Movement happens in this space as learning proceeds. For example, when solving a puzzle in The Incredible Machine, a student gets a bright idea how to implement a solution and then builds its simulation. If she runs the simulation and it fails, she sees that her idea has some part that doesn't work - that needs to be diagnosed and reconstructed. At this point the student may move down into the lower half of the diagram (Quadrant III) into the 'dark teatime of the soul' while discarding misconceptions and unproductive ideas. As she consolidates her knowledgewhat works and what does not-with awareness of a sense of making progress, she advances to Quadrant IV. Getting another fresh idea propels the student back into the upper half of the space (Quadrant I). Thus, a typical learning experience involves a range of emotions, cycling the student around the four quadrant cognitive-emotive space as they learn.

If one visualizes a version of Figure 4A (and Figure 4B) for each axis in Figure 3, then at any given instant, the student might be in multiple Quadrants with respect to different axes. They might be in Quadrant II with respect to feeling frustrated and simultaneously in Quadrant I with respect to interest level. It is important to recognize that a range of emotions occurs naturally in a real learning process, and it is not simply the case that the positive emotions are the good ones.

We do not foresee trying to keep the student in Quadrant I, but rather to help him see that the cyclic nature is natural in learning science, technology, engineering, or mathematics (STEM), and that when he lands in the negative half, it is an inevitable part of the cycle. Our aim is to help students to keep orbiting the loop, teaching them to propel themselves, especially after a setback.
A third axis (not shown) can be envisioned as extending out of the plane of the page-the cumulative knowledge axis. If one visualizes the above dynamics of moving from Quadrant I to II to III to IV as an orbit, then, when this third dimension is added, one obtains an excelsior spiral. In Quadrant I, anticipation and expectation are high, as the learner builds ideas and concepts and tries them out. Emotional mood decays over time either from boredom or from disappointment. In Quadrant II, the rate of construction of working knowledge diminishes, and negative emotions emerge as progress wanes. In Quadrant III, as the negative affect runs its course, the learner discards misconceptions and ideas that didn't pan out. In Quadrant IV, the learner recovers hopefulness and positive attitude as the knowledge set is now cleared of unworkable and unproductive concepts, and the cycle begins anew. In building a complete and correct mental model associated with a learning opportunity, the learner may experience multiple cycles until completion of the learning exercise. Note that the orbit doesn't close on itself, but gradually spirals around the cumulative knowledge axis.

These models suggest constructive ways for an educator to intervene more artfully as a teacher, coach, or mentor striving to enliven and enrich the learning journey in more emotionally satisfying ways. In particular the FourQuadrant model (Figure 4A) suggests distinct intervention strategies appropriate to each Quadrant (e.g., supporting diagnosis of misconceptions in Quadrant II to forestall frustration).

\section{CONCLUSION}

Our models are inspired by theory often used to describe complex dynamic interactions in engineering systems. As such, they are not intended to explain how learning works, but rather to provide a framework for thinking and posing questions about the role of emotions in learning. As with any metaphor, the model has its limits. The model does not encompass all aspects of the complex interaction between 
emotions and learning, but begins to describe some of the key phenomena that needs to be considered in metacognition.

These models go beyond previous research studies not just in the range of emotions addressed, but also in an attempt to formalize an analytical model that describes the dynamics of a learner's emotional states, and does so in a language that supports metacognitive analysis.

\section{ACKNOWLEDGEMENT}

This material is based upon work supported by the National Science Foundation under Grant No. 0087768. Any opinions, findings, or conclusions or recommendations expressed in this material are those of the author(s) and does not necessarily reflect the views of the National Science Foundation.

\section{REFERENCES}

[1] Goleman, D., (1995). Emotional Intelligence. Bantam Books: New York.

[2] Csikszentmihalyi, M. (1990). Flow: The Psychology of Optimal Experience, Harper-Row: NY.

[3] Picard, Rosalind. (2000). Toward Computers that Recognize and Respond to User Emotions, IBM Systems Journal, Vol. 39 (3 and 4), p. 705 .

[4] Scheirer, J., Fernandez, R., P icard, R. (1999), Expression Glasses: A Wearable Device for Facial Expression Recognition, Proceedings of CHI, February 1999.

[5] Bartlett, M., Hager, J.C., Ekman, P., and Sejnowski, T. (1999). Measuring Facial Expression by Computer Image Analysis. Psychophysiology, vol. 36, pp. 253-263.

[6] Cohn, J.F., Zerlochow, J., Lien, J., and Kanade, T. (1999). Automated Face Analysis by Feature Point Tracking has High Concurrent Validity with Manual FACS Coding, Psychophysiology, vol. 36, pp35-43.

[7] Desilva, L.C., Miyasato, T., and Nakatsu, R., Facial emotion recognition using multimodal information, in Proc. IEEE Int. Conf. on Info., Comm. and Sig. Proc., (Singapore), pp. 397-401, Sept 1997.

[8] Donato, G., Bartlett, M.S., Hager, J.C., Ekman, P., and Sejnowski, T.J., Classifying facial actions, IEEE Pattern Analy. and Mach. Intell., vol. 21, pp. 974--989, October 1999.

[9] Ekman, Paul. (1997). Facial Action Coding System, Consulting Psychologists Press.

[10] Essa, I. and Pentland, A., Coding, analysis, interpretation and recognition of facial expressions, IEEE Transactions on Pattern Analysis and Machine Intelligence, vol. 19, pp. 757--763, July 1997.

[11] Chen, L.S., Huang, T.S., Miyasato, T., and Nakatsu, R. (1998). Multimodal Human Emotion/Expression Recognition. Proceedings of 3rd International Conference on Automated Face and Gesture Recognition, pp366-371.

[12] Huang, T.S., Chen, L.S., and Toa, H. (1998). Bimodal Emotion Recognition by Man and Machine. ATR Workshop on Virtual Communication Environments.

[13] Kapoor, A., Mota, S. and Picard, R. (2001). Towards a Learning Companion that Recognizes Affect, Proceedings of AAAI 2001.

0-7803-7444-4/02/\$17.00 @ 2002 IEEE

November 6 - 9, 2002, Boston, MA

$32^{\text {nd }}$ ASEE/IEEE Frontiers in Education Conference 